\title{
Association between serum uric acid and renal outcome in patients with biopsy-confirmed diabetic nephropathy
}

\author{
Yutong Zou ${ }^{1,2}$, Lijun Zhao ${ }^{1,2}$, Junlin Zhang ${ }^{1,2}$, Yiting Wang ${ }^{1,2}$, Yucheng $\mathrm{Wu}^{1,2}$, Honghong Ren ${ }^{1,2}$, Tingli Wang ${ }^{1,2}$, \\ Rui Zhang ${ }^{1,2}$, Jiali Wang ${ }^{1,2}$, Yuancheng Zhao ${ }^{1,2}$, Chunmei Qin ${ }^{1,2}$, Huan $\mathrm{Xu}^{3}$, Lin $\mathrm{Li}^{3}$, Zhonglin Chai ${ }^{4}$, Mark E Cooper ${ }^{4}$, \\ Nanwei Tong ${ }^{5,6}$ and Fang Liu(1) 1,2 \\ 1'Division of Nephrology, West China Hospital of Sichuan University, Chengdu, Sichuan, China \\ 'Laboratory of Diabetic Kidney Disease, Centre of Diabetes and Metabolism Research, West China Hospital of Sichuan University, Chengdu, \\ Sichuan, China \\ ${ }^{3}$ Division of Pathology, West China Hospital of Sichuan University, Chengdu, Sichuan, China \\ ${ }^{4}$ Department of Diabetes, Central Clinical School, Monash University, Melbourne, Australia \\ ${ }^{5}$ Division of Endocrinology, West China Hospital of Sichuan University, Chengdu, Sichuan, China \\ ${ }^{6}$ Centre of Diabetes and Metabolism Research, West China Hospital of Sichuan University, Chengdu, Sichuan, China
}

\begin{abstract}
Objective: To investigate the relationship between serum uric acid (SUA) level and renal outcome in patients with type 2 diabetes mellitus (T2DM) and diabetic nephropathy (DN). Methods: A total of 393 Chinese patients with T2DM and biopsy-proven DN and followed at least 1 year were enrolled in this study. Patients were stratified by the quartiles of baseline level of SUA: Q1 group: $286.02 \pm 46.66 \mu \mathrm{mol} / \mathrm{L}(n=98)$; Q2 group: $358.23 \pm 14.03 \mu \mathrm{mol} / \mathrm{L}(n=99) ;$ Q3 group: $405.50 \pm 14.59 \mu \mathrm{mol} / \mathrm{L}(n=98)$ and Q4 group: $499.14 \pm 56.97 \mu \mathrm{mol} / \mathrm{L}(n=98)$. Renal outcome was defined by progression to end-stage renal disease (ESRD). Kaplan-Meier survival analysis and Cox proportional hazards model were used to analyze the association between SUA quartiles and the renal outcomes. Results: During the median 3-year follow-up period, there were 173 ESRD outcome events (44.02\%). No significant difference between SUA level and the risk of progression of DN $(P=0.747)$ was shown in the Kaplan-Meier survival analysis. In multivariableadjusted model, hazard ratios for developing ESRD were $1.364(0.621-2.992 ; P=0.439)$, $1.518(0.768-3.002 ; P=0.230)$ and $1.411(0.706-2.821 ; P=0.330)$ for the Q2, Q3 and Q4, respectively, in comparison with the Q1 $(P=0.652)$.

Conclusions: No significant association between SUA level and renal outcome of ESRD in Chinese patients with T2DM and DN was found in our study. Besides, the role of uric acidlowering therapy in delaying DN progression and improving ESRD outcome had not yet been proven. Further study was needed to clarify the renal benefit of the uric acid-lowering therapy in the treatment of DN.
\end{abstract}
Key Words
- serum uric acid
- renal outcome
- type 2 diabetes mellitus
- diabetic nephropathy

\section{Introduction}

It is well established that diabetic nephropathy (DN) is the first cause of end-stage renal disease (ESRD) in developed and developing countries (1). In China, both the incidence and prevalence of DN have risen dramatically over the last decade. In China Kidney Disease Network 2015 annual data report, diabetes-related kidney disease is now the leading cause of chronic kidney disease (CKD), accounting for $26.96 \%$ (2).

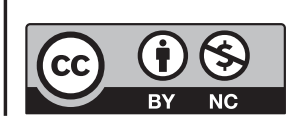


Serum uric acid (SUA) level has been widely recognized as a risk factor for DN development because oxidants are produced that can play a key role in renal injury during uric acid synthesis (3). A host of previous studies had reported an association between SUA levels and DN development. It is generally accepted that hyperuricemia was considered to be a risk factor independent of other indicators for the development of incident chronic kidney disease (CKD) in type 2 diabetic mellitus (T2DM) patients with preserved kidney function $(4,5)$. Furthermore, the association among SUA, reduction of SUA and DN progression in T2DM is still controversial. Study of Hayashino et al. suggested the outcomes of progression from microalbuminuria to macroalbuminuria as well as change from normoalbuminuria to microalbuminuria or macroalbuminuria, showing that SUA level was correlated to albuminuria progression, not albuminuria development in T2DM patients in Japan (6). Besides, a study by Chang et al. suggested that the SUA level was correlated to CKD regression and progression. Besides, for T2DM patients with stage 3-5 CKD, a lower level of SUA could be beneficial for the improvement of CKD. A high-normal SUA level was also suggested to require careful monitoring in Taiwan T2DM patients (7). Therefore, SUA levels might be a good factor in predicting the risk of progression of DKD. It is still insufficient to recommend the addition of SUA-lowering treatment to the care standard for diabetic patients who had a higher risk of diabetic kidney disease (DKD), although previous studies indicated a prospective benefit of SUA-lowering treatment on progression of kidney disease (8). Recently, a randomized controlled trial (RCT) showed that in multi-ethnic patients with a high risk of progression and stage 3-4 CKD in Australia and New Zealand, SUA-lowering treatment using allopurinol could not significantly attenuate the drop of eGFR as compared with placebo (9), which is somewhat intriguing. Taken together, the association between SUA and renal outcome in T2DM and $\mathrm{DN}$ patients remains to be elucidated in more detail.

The purpose of this study was to determine whether SUA levels can predict the renal outcome of biopsy-proven DN in patients with T2DM.

\section{Methods}

\section{Study population and design}

A total of $393 \mathrm{~T} 2 \mathrm{DM}$ and DN patients who had undergone percutaneous renal biopsy between January 2008 and July 2019 and had at least 1-year follow-up at the West China
Hospital of Sichuan University were enrolled for the longitudinal observational study. T2DM was diagnosed in accordance with the American Diabetes Association criteria. The renal biopsy indications were renal damage and T2DM, without absolute contraindication (10). DN was diagnosed by at least two renal pathologists and/or nephrologists on the basis of the Renal Pathology Society classification and was defined using the standard of An et al. in $2015(11,12)$. The inclusion criteria were patients (1) with a diagnosis of T2DM, (2) aged $\geq 18$ years old, (3) with a diagnosis of renal biopsy-proven $\mathrm{DN}$ and (4) with an e-GFR $>15 \mathrm{~mL} / \mathrm{min} / 1.73 \mathrm{~m}^{2}$. The exclusion criteria were participants with non-T2DM, a history of kidney transplantation or incomplete data, those who began the dialysis treatment before the renal biopsy and those with a follow-up time of less than 1 year (Fig. 1). All the patients undergone renal biopsies gave signed consent. Patients were stratified by the quartiles of baseline level of SUA as Q1, Q2, Q3 and Q4 group.

\section{Clinical and laboratory characteristics}

The following baseline clinical and laboratory characteristics were collected: age, sex, blood urea nitrogen (BUN), 24-h urine: urinary total protein (UTP), serum creatinine (sCr), serum albumin (sAlb), SUA, systolic/ diastolic blood pressure (SBP/DBP), the duration of diabetes, the presence of diabetic retinopathy (DR), LDL-C, HDL-C, total cholesterol, glycosylated hemoglobin (HbA1c), hemoglobin $(\mathrm{Hb})$, cystatin-C (cys-C), the estimated glomerular filtration rate (eGFR), calculated using the equation of the CKD-EPI and the use of insulin, metformin,

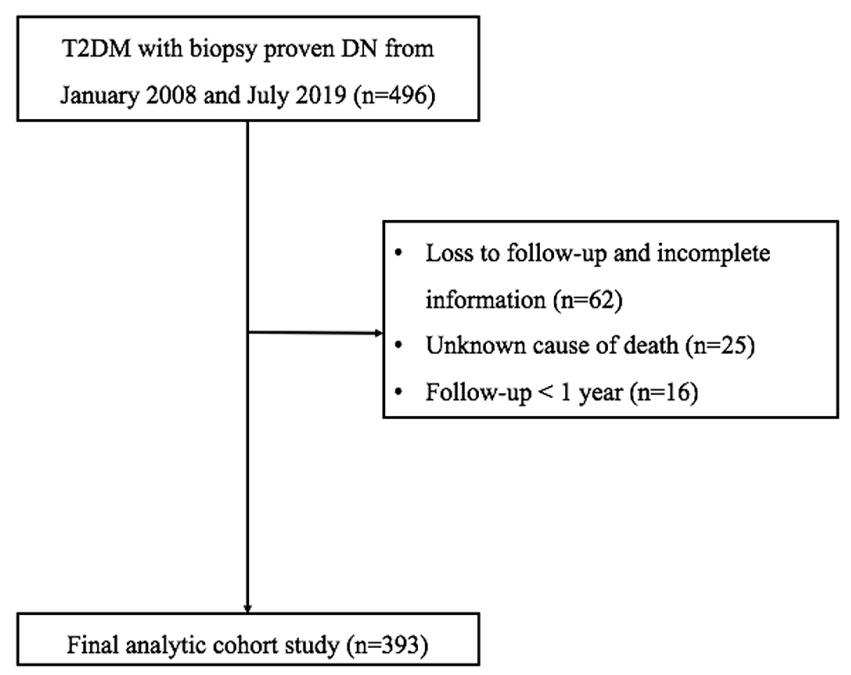

Figure 1

Flowchart for recruitment procedures of the predictive model for ESRD. 
diuretics, calcium channel blockers (CCB), angiotensinconverting enzyme inhibitors (ACEI), angiotensin receptor blocker (ARB), statins and antihyperuricemic drug. The trajectories of eGFR decline and SUA change were evaluated using a simple linear model. Moreover, hyperuricemia was defined as SUA higher than $420 \mu \mathrm{mol} / \mathrm{L}$ in men and $360 \mu \mathrm{mol} / \mathrm{L}$ in women.

\section{Renal biopsy-related information}

The pathological classifications of glomerular alterations, interstitial fibrosis and tubular atrophy (IFTA), interstitial inflammation and arteriolar hyalinosis were on the basis of the criteria published by the Renal Pathology Society (12). The pathological lesions were graded by at least two pathologists.

\section{Outcome definition}

The renal outcome was progression to ESRD, defined by commencement of renal replacement therapy or an eGFR $<15 \mathrm{~mL} / \mathrm{min} / 1.73 \mathrm{~m}^{2}$ (11). Death was not included with ESRD as an event.

\section{Statistics}

For continuous variables, data were presented as the mean \pm S.D. or median with range. Categorical variables were presented as ratios. A one-way ANOVA was used to evaluate differences in means among groups. Chisquared or Fisher's exact tests were used to compare the categorical variables. Kaplan-Meier survival analysis and Cox proportional hazards model were used to analyze the association between SUA quartiles and the renal outcomes. Three statistical models were used in analysis: the crude model; the second model was adjusted for age and sex; and the third model was adjusted for age, sex, eGFR, UTP, sAlb, HbA1c, total cholesterol, the use of antihyperuricemic drug, glomerular class, IFTA, interstitial inflammation and arteriolar hyalinosis. All statistical tests were analyzed using SPSS version 25.0.

\section{Results}

\section{Clinical and pathological baseline characteristics of the total participants}

The baseline clinical and pathological data of the 393 patients were reported in Table 1 . During the median 3-year follow-up period, there were 173 ESRD outcome events $(44.02 \%)$, which is consistent with other studies $(13,14,15)$. The mean patient age was $51.06 \pm 9.39$ years old, and males comprised $71 \%$. The mean SUA was $387.15 \pm 86.21 \mu \mathrm{mol} / \mathrm{L}$. The mean sAlb level was $34.46 \pm 7.73 \mathrm{~g} / \mathrm{L}$, and the mean Hb level was $120.05 \pm 4.49 \mathrm{~g} / \mathrm{L}$. The mean sCr level was $136.46 \pm 82.23 \mu \mathrm{mol} / \mathrm{L}$ and mean e-GFR was $66.30 \pm 31.85 \mathrm{~mL} / \mathrm{min} / 1.73 \mathrm{~m}^{2}$. The percentage of participants who were complicated with DR was $44.53 \%$. The percentage of participants who had cardio/cerebrovascular diseases, heart failure and gouty arthritis were 38.5, 7.3 and $2.8 \%$, respectively. Patients were categorized by the quartiles of baseline level of SUA: Q1 group $286.02 \pm 46.66 \mu \mathrm{mol} / \mathrm{L}(n=98) ;$ Q2 group: $358.23 \pm 14.03 \mu \mathrm{mol} / \mathrm{L}(n=99) ; \mathrm{Q} 3$ group: $405.50 \pm 14.59$ $\mu \mathrm{mol} / \mathrm{L}(n=98)$ and Q4 group: $499.14 \pm 56.97 \mu \mathrm{mol} / \mathrm{L}$ $(n=98)$. The percentage of patients progressed to ESRD during the follow-up period in the four groups was 43.88 , $45.45,43.88$ and $42.86 \%$, respectively.

As for the pathological characteristics, the consumption of glomerular classification of class I, IIa, IIb, III and IV was 5.1, 22.4, 14.2, 43.5 and $14.8 \%$, respectively. IFTA scores of $0,1,2$ and 3 accounted for 2.5, 44.3, 40.2 and $13.0 \%$, respectively. Interstitial inflammation scores of 0,1 and 2 consisted of 5.3, 72.8 and 21.9\%, respectively. Arteriolar hyalinosis scores of 0, 1 and 2 were occupied 9.9, 48.3 and $41.7 \%$ patients, respectively.

When it comes to the drug using, $21.05 \%$ of patients used antihyperuricemic drugs, $10.43 \%$ of patients used ACEI, $71.76 \%$ of patients used ARB, $66.7 \%$ of patients used CCB, $22.1 \%$ of patients used diuretics, $58.8 \%$ of patients used statins and $29.0 \%$ of patients used beta-blockers.

\section{Baseline clinical and pathologic characteristics among the four groups stratified by the quartiles of baseline level of SUA}

There was no significant difference observed in age, $\mathrm{Hb}$, diabetes duration, phosphate, calcium, fasting glucose, total cholesterol, triglyceride (TG), HDL-C, SBP, DBP, cys-C, history of smoking, family history of DM, DR, glomerular class, IFTA, interstitial inflammation, arteriolar hyalinosis, use of ACEI, use of ARB, use of $\mathrm{CCB}$, use of statins and use of beta-blockers among the four groups stratified by the quartiles of baseline level of SUA. Noticeably, participants with a higher level of SUA were prone to be males $(P<0.001)$ and have higher sAlb $(P<0.001)$, higher $\mathrm{sCr}(P=0.018)$, lower HbA1c $(P=0.020)$, higher BUN $(P=0.004)$, lower eGFR $(P=0.023)$, lower LDL-C $(P=0.004)$, lower UTP (0.046), higher percentage of gouty arthritis (0.018),

This work is licensed under a Creative Commons Attribution-NonCommercial 4.0 International License. 
Table 1 Baseline characteristics of the study population.

\begin{tabular}{|c|c|c|c|c|c|c|}
\hline & \multirow[b]{2}{*}{$\begin{array}{c}\text { All subjects, } \\
n=393\end{array}$} & \multicolumn{4}{|c|}{ Serum uric acid quartiles } & \multirow[b]{2}{*}{ P value } \\
\hline & & $\begin{array}{c}\text { First quartile, } \\
\quad n=98\end{array}$ & $\begin{array}{c}\text { Second quartile, } \\
n=99\end{array}$ & $\begin{array}{c}\text { Third quartile, } \\
n=98\end{array}$ & $\begin{array}{c}\text { Fourth quartile, } \\
n=98\end{array}$ & \\
\hline Serum uric acid ( $\mu \mathrm{mol} / \mathrm{L})$ (mean (s.D.)) & $387.15(86.21)$ & $286.02(46.66)$ & $358.23(14.03)$ & $405.50(14.59)$ & $499.14(56.97)$ & $<0.001$ \\
\hline Age, years & 51.06 (9.39) & $51.35(9.75)$ & $52.02(8.54)$ & $51.79(9.80)^{a}$ & $49.96(9.67)$ & 0.531 \\
\hline Sex, male, n (\%) & 279 (70.99) & $55(56.1)$ & $67(67.7)$ & $70(71.4)$ & $87(88.8)^{a, b, c}$ & $<0.001$ \\
\hline Diabetes duration, months & 99.93 (69.77) & $95.74(69.16)$ & $101.85(64.69)$ & $96.95(60.10)$ & $104.32(86.41)$ & 0.997 \\
\hline Serum albumin (g/L) & $34.46(7.73)$ & $30.93(6.64)$ & $33.57(7.81)$ & $35.97(7.85)$ & $37.26(7.21)$ & $<0.001$ \\
\hline $\mathrm{Hb}(\mathrm{g} / \mathrm{L})$ & $120.05(4.49)$ & $114.68(31.61)$ & $124.57(30.14)$ & $121.48(25.00)$ & $119.48(25.05)$ & 0.492 \\
\hline Phosphate (mg/dL) & $1.21(0.25)$ & $1.22(0.27)$ & $1.20(0.24)$ & $1.19(0.25)$ & $1.21(0.26)$ & 0.912 \\
\hline Calcium (mmol/L) & $2.14(0.17)$ & $2.11(0.19)$ & $2.14(0.15)$ & $2.15(0.15)$ & $2.17(0.17)$ & 0.260 \\
\hline Fasting glucose (mmol/L) & $8.42(4.23)$ & $8.74(4.28)$ & $8.60(4.88)$ & $8.35(4.10)$ & $7.74(3.96)^{a, b}$ & 0.151 \\
\hline $\mathrm{HbA} 1 \mathrm{c}(\%)$ & $7.58(1.96)$ & $8.03(2.48)$ & $7.58(1.95)^{b}$ & $7.68(1.81)^{b}$ & $7.08(1.58)^{b}$ & 0.020 \\
\hline Total cholesterol (mmol/L) & $5.21(1.67)$ & $5.39(1.74)$ & $5.31(1.66)$ & $5.12(1.50)^{b}$ & $5.02(1.58)$ & 0.132 \\
\hline Triglyceride (mmol/L) & $2.20(1.71)$ & 2.13 (1.19) & $1.98(1.14)$ & $2.42(2.35)^{b}$ & $2.57(2.33)^{a, b}$ & 0.056 \\
\hline LDL-C (mmol/L) & $3.04(1.33)$ & $3.29(1.44)$ & $3.20(1.42)^{b}$ & $2.83(1.15)^{b}$ & $2.83(1.12)^{b}$ & 0.004 \\
\hline HDL-C (mmol/L) & $1.37(0.62)$ & $1.32(0.48)$ & $1.36(0.47)$ & $1.37(0.65)$ & $1.19(0.43)$ & 0.237 \\
\hline eGFR (mL/min/1.73 $\left.\mathrm{m}^{2}\right)$ & $66.30(31.85)$ & $67.15(31.00)$ & $74.87(35.64)$ & $63.08(27.35)^{a}$ & $59.31(30.27)$ & 0.023 \\
\hline $\mathrm{sCr}(\mu \mathrm{mol} / \mathrm{L})$ & $136.46(82.23)$ & $132.31(84.10)$ & $122.73(73.88)$ & $134.41(66.77)$ & $159.74(103.97)$ & 0.018 \\
\hline 24-h urine: urinary total protein, g/24 h & $5.32(4.49)$ & $6.30(4.68)$ & $4.77(4.21)$ & $4.46(3.90)$ & $4.45(3.71)$ & 0.046 \\
\hline $\mathrm{SBP}(\mathrm{mmHg})$ & $145.57(23.07)$ & $148.40(21.35)$ & $148.28(23.18)$ & $146.02(23.29)$ & $143.85(24.30)$ & 0.932 \\
\hline DBP (mmHg) & $86.17(13.23)$ & $87.38(13.84)$ & 84.55 (12.12) & $85.57(14.42)^{a}$ & $85.71(13.02)$ & 0.474 \\
\hline BUN (mg/dL) & $8.79(3.94)$ & $8.53(3.64)$ & $7.68(3.92)$ & $9.29(3.93)$ & $9.64(4.49)$ & 0.004 \\
\hline Cystatin-C (mg/L) & $1.70(0.91)$ & $1.83(1.32)$ & $1.55(0.63)^{b}$ & $1.62(0.52)^{b}$ & $1.75(0.76)$ & 0.295 \\
\hline Use of antihyperuricemic drugs (\%) & 21.05 & 6.7 & 13.6 & $27.3^{\mathrm{b}}$ & $33.8^{\mathrm{b}, \mathrm{a}}$ & $<0.001$ \\
\hline Use of ACEI (\%) & 10.43 & 13.3 & 6.1 & 10.2 & 12.2 & 0.359 \\
\hline Use of ARB (\%) & 71.76 & 78.6 & 71.7 & 71.4 & 65.3 & 0.234 \\
\hline Use of CCB (\%) & 66.7 & 66.3 & 64.6 & 67.3 & 68.4 & 0.953 \\
\hline Use of diuretics (\%) & 22.1 & 35.7 & $18.2^{b}$ & $13.3^{b}$ & $21.4^{b, c}$ & $<0.001$ \\
\hline Use of loop-acting diuretics (\%) & 18.3 & 27.0 & 17.2 & 13.0 & 16.2 & 0.063 \\
\hline Use of potassium-sparing diuretics (\%) & 4.8 & 8.0 & 3.0 & 2.0 & 6.1 & 0.171 \\
\hline Use of thiazide diuretics (\%) & 3.3 & 7.0 & 2.0 & 1.0 & 3.0 & 0.088 \\
\hline Use of statins (\%) & 58.8 & 64.3 & 58.6 & 54.1 & 58.2 & 0.545 \\
\hline Use of beta-blockers (\%) & 29.0 & 29.6 & 32.3 & 25.5 & 28.6 & 0.768 \\
\hline History of smoking (\%) & 47.33 & 43.9 & 46.5 & 44.9 & 54.1 & 0.471 \\
\hline Family history of DM (\%) & 31.81 & 30.6 & 25.3 & 36.7 & 34.7 & 0.321 \\
\hline Cardio/cerebrovascular diseases (\%) & 38.5 & 35.0 & 47.5 & 35.0 & 36.4 & 0.204 \\
\hline Heart failure (\%) & 7.3 & 7.0 & 7.1 & 8.0 & 7.1 & 0.992 \\
\hline Gouty arthritis (\%) & 2.8 & 0 & 2.0 & 2.0 & 7.1 & 0.018 \\
\hline Diabetic retinopathy (\%) & 44.53 & 52.0 & 42.4 & 42.9 & 40.8 & 0.380 \\
\hline \multicolumn{7}{|l|}{ Pathological parameters } \\
\hline \multicolumn{7}{|l|}{ Glomerular class (\%) } \\
\hline I & 5.1 & 5.1 & 5.1 & 4.1 & 6.1 & 0.433 \\
\hline Ila & 22.4 & 17.3 & 23.2 & 27.6 & 21.4 & \\
\hline Ilb & 14.2 & 16.3 & 10.1 & 17.3 & 13.3 & \\
\hline III & 43.5 & 48.0 & 50.5 & 37.8 & 37.8 & \\
\hline IV & 14.8 & 13.3 & 11.1 & 13.3 & 21.4 & \\
\hline \multicolumn{7}{|l|}{ IFTA (\%) } \\
\hline 0 & 2.5 & 4.1 & 1.0 & 3.1 & 2.0 & 0.529 \\
\hline 1 & 44.3 & 51.0 & 45.5 & 44.9 & 35.7 & \\
\hline 2 & 40.2 & 33.7 & 42.4 & 38.8 & 45.9 & \\
\hline 3 & 13.0 & 11.2 & 11.1 & 13.3 & 16.3 & \\
\hline \multicolumn{7}{|l|}{ Interstitial inflammation (\%) } \\
\hline 0 & 5.3 & 3.1 & 6.1 & 9.2 & 3.1 & 0.348 \\
\hline 1 & 72.8 & 78.6 & 69.7 & 67.3 & 75.5 & \\
\hline 2 & 21.9 & 18.4 & 24.2 & 23.5 & 21.4 & \\
\hline \multicolumn{7}{|l|}{ Arteriolar hyalinosis (\%) } \\
\hline 0 & 9.9 & 8.2 & 6.1 & 12.2 & 13.3 & 0.299 \\
\hline 1 & 48.3 & 49.0 & 44.4 & 46.9 & 53.1 & \\
\hline 2 & 41.7 & 42.9 & 49.5 & 40.8 & 33.7 & \\
\hline
\end{tabular}

${ }^{a} P<0.05$ vs the $2 Q$ group; ${ }^{b P}<0.05$ vs the $1 Q$ group; ${ }^{c} P<0.05$ vs the $3 Q$ group.

BUN, blood urea nitrogen; DBP: diastolic blood pressure; eGFR, estimated glomerular filtration rate; Hb: hemoglobin; IFTA, interstitial fibrosis and tubular atrophy; SBP, systolic blood pressure; sCr, serum creatinine; UA, uric acid.

https://ec.bioscientifica.com

https://doi.org/10.1530/EC-21-0307 (c) 2021 The authors Published by Bioscientifica Ltd
$10: 10$

12 
higher use of antihyperuricemic drug $(P<0.001)$ and lower use of diuretics $(P<0.001)$. Besides, in contrast to patients in the Q2 group, eGFR was significant lower in Q3 group. Q3 and Q4 groups showed significant higher TG level compared with Q1 group. Total cholesterol and cys-C level was significant lower in Q3 group compared with Q1 group. No significance was found in pathological parameters containing glomerular class, interstitial inflammation, IFTA and arteriolar hyalinosis among four groups.

Correlations between the level of SUA and clinicalhistopathological findings were shown in Table 2. The results showed that SUA level had a significant positive correlation with IFTA score $(P=0.011)$ and a negative correlation with e-GFR $(P<0.001)$ when adjusting for age and sex. Moreover, the level of SUA was shown to have a significant positive correlation with TG $(P<0.001)$ and sAlb $(P<0.001)$ when adjusting for age, sex and e-GFR, as well as a negative correlation with UTP $(P<0.001)$ and HbA1c $(P=0.040)$ when adjusting for age, sex and e-GFR.

\section{SUA level and renal outcome among the four groups stratified by the quartiles of baseline level of SUA}

Kaplan-Meier survival analysis showed no significant difference in renal survival rate among the four groups stratified by the quartiles of baseline level of SUA $(P=0.747)$ (Fig. 2). Besides, Kaplan-Meier survival analysis of the renal survival rate in DN patients with or without hyperuricemia showed no significant difference as well $(P=0.535)$ (Fig. 3).

Table 3 displays the clinicopathological features of participants in cox progression in conformity with the SUA quartiles. The hazard ratios (HRs) of crude model for the progression of DN were 0.859 (95\% CI: 0.564-1.308; $P=0.480), 0.872(0.571-1.332 ; P=0.526)$ and $0.791(0.517-$ 1.211; $P=0.281$ ) for the $\mathrm{Q} 2, \mathrm{Q} 3$ and $\mathrm{Q} 4$, respectively, as compared with that for Q1 $(P=0.753)$. In age- and sexadjusted model, HRs for developing ESRD were 0.865 (0.566-1.322; $P=0.502), 0.875$ (0.572-1.340; $P=0.540)$ and $0.777(0.501-1.204 ; P=0.259)$ for Q2, Q3 and Q4, respectively, in comparison with the Q1 $(P=0.732)$. In multivariable-adjusted model, HRs for developing ESRD were 1.364 (0.621-2.992; $P=0.439), 1.518(0.768-3.002$; $P=0.230)$ and $1.411(0.706-2.821 ; \mathrm{p}=0.330)$ for the Q2, Q3 and Q4, respectively, in comparison with the $\mathrm{Q} 1(P=0.652)$.

In addition, the annual decline in eGFR and SUA is calculated in 102 patients with the record of SUA and eGFR $\geqq 3$ times. The median eGFR slope is $-6.8 \mathrm{~mL} / \mathrm{min} / 1.73$ $\mathrm{m}^{2} /$ year. There is no significant correlation between the annual decline in eGFR and baseline SUA $(P=0.072)$, mean SUA during follow-up $(P=0.635)$ as well as the annual decline in SUA $(P=0.080)$ after adjusting the age and sex. The distribution of the annual change in eGFR and SUA in the patients is shown in the Supplementary Materials (see section on supplementary materials given at the end of this article).

This result suggested that SUA levels might not be correlated with the risk of DN progression.

\section{Discussion}

In this study, we explored the relationship between the SUA levels and clinicopathological characteristics and renal outcomes in 393 Chinese T2DM patients with biopsyproven DN. No significant association was found between SUA levels and renal outcome. Noticeably, lower SUA group had lower sAlb, less males, higher HbA1c, higher LDL-C, UTP, which might indicate that the baseline metabolic condition could be worse in lower SUA group than higher one. Moreover, $21.05 \%$ of patients used antihyperuricemic drugs, $10.43 \%$ used ACEI, $71.76 \%$ used ARB, $66.7 \%$ used CCB, $22.1 \%$ used diuretics, $58.8 \%$ used statins and $29.0 \%$ used beta-blockers. Patients with higher uric acid levels tended to have higher use of antihyperuricemic drugs and lower use of diuretics. Generally, these drugs could also affect SUA levels and might have an impact on outcomes during the period $(16,17)$.

Table 2 Correlations between the serum uric acid level and clinical-histopathological findings.

\begin{tabular}{|c|c|c|c|}
\hline & Variables & Correlation coefficient $(r)$ & P value \\
\hline \multirow[t]{6}{*}{ Serum uric acid } & IFTA & 0.127 & $0.011^{a}$ \\
\hline & $\mathrm{e}-\mathrm{GFR}\left(\mathrm{mL} / \mathrm{min} / 1.73 \mathrm{~m}^{2}\right)$ & -0.165 & $0.001^{\mathrm{b}}$ \\
\hline & 24-h urine: urinary total protein (g/day) & -0.186 & $0.001^{c}$ \\
\hline & $\mathrm{HbA} 1 \mathrm{c}(\%)$ & -0.116 & $0.040^{c}$ \\
\hline & Triglyceride (mmol/L) & 0.174 & $0.001^{c}$ \\
\hline & Serum albumin (g/L) & 0.351 & $0.001^{c}$ \\
\hline
\end{tabular}

aSpearman's correlation analysis. A two-tailed $P<0.05$ was considered statistically significant; ${ }^{b}$ partial correlation analysis for adjusting the baseline age and sex; ' partial correlation analysis for adjusting the baseline age, sex and e-GFR.

IFTA: interstitial fibrosis and tubular atrophy.

https://ec.bioscientifica.com

https://doi.org/10.1530/EC-21-0307 (c) 2021 The authors Published by Bioscientifica Ltd

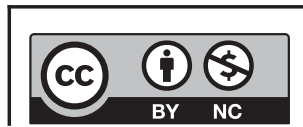

This work is licensed under a Creative Commons Attribution-NonCommercial 4.0 International License. 


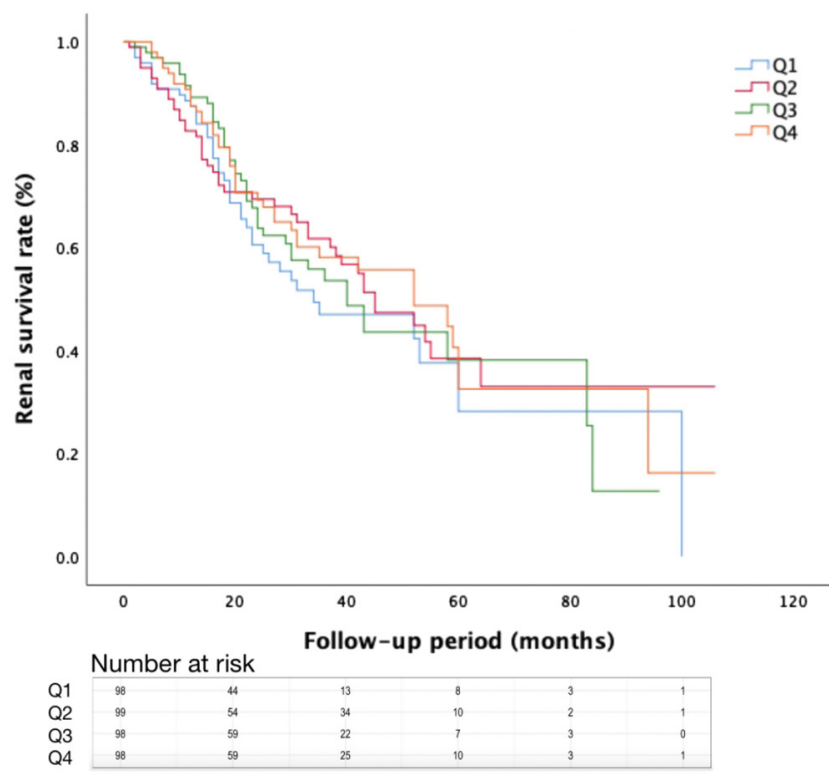

Figure 2

Kaplan-Meier curves of the renal survival rate in DN patients with different serum uric acid levels.

It seemed that SUA was a double-edged sword. On the one hand, it constitutes the most crucial natural antioxidant, providing up to $60 \%$ of the body's free radical scavenging capacity (18). But on the other hand, long-term high uric acid exposure can induce gout and renal damage. In pre-clinical studies, SUA has been proven to elicit nitric oxide pathway alteration, to activate the renin-angiotensin system and to induce pro-inflammatory cytokines (19). Several lines of evidence suggest an association between SUA and kidney disease, showing that hyperuricemia

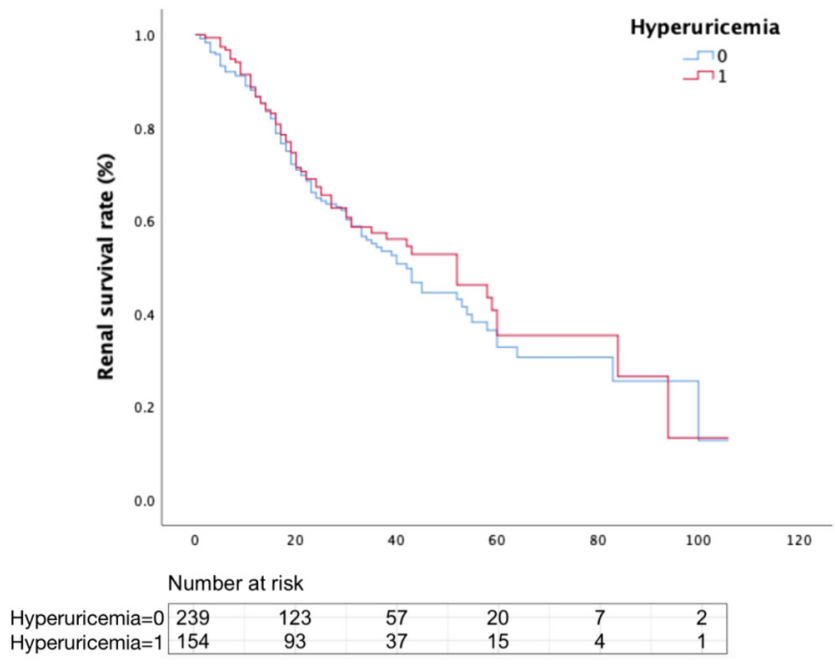

Figure 3

Kaplan-Meier curves of the renal survival rate in DN patients with or without hyperuricemia. is closely related to kidney disease, and long-term hyperuricemia could lead to damage and deterioration of renal function $(20,21)$.

So far, however, there has been little discussion about the association of SUA and renal outcome in T2DM and DN patients. Increased SUA levels have also been correlated with DN progression among patients who have already developed this complication. Hayashino et al. suggested the outcomes of progression from microalbuminuria to macroalbuminuria as well as change from normoalbuminuria to microalbuminuria or macroalbuminuria in a 2-year follow-up study of 2518 T2DM patients (19). In this study, the baseline eGFR was $77.4 \pm 19.0 \mathrm{~mL} / \mathrm{min} / 1.73 \mathrm{~m}^{2}, 14.7 \%$ of patients had history of cardiovascular disease as well as $42.2 \%$ of participants had diabetic retinopathy. The means of SUA were 3.6, 4.9, 5.8 and $7.3 \mathrm{mg} / \mathrm{dL}$ for the quartile 1 to quartiles 4 , respectively. In addition, a U-shaped risk curve for progression from microalbuminuria to macroalbuminuria was found in their study. The group whose UA concentration was $4.8 \mathrm{mg} / \mathrm{dL}$ (interquartile range (IQR): $4.6-5.0 \mathrm{mg} / \mathrm{dL}$ ) showed the lowest risk of DN progression. Although the association between higher level of SUA and DN progression had been expected, but the higher HR in lowest SUA quartile remained unexplained. Moreover, no significant association of SUA with eGFR change or with progression from normalbuminuria to microalbuminuria was found which might be because of the short study duration (the median 1.8-year follow-up) and relatively benign kidney function. Noticeably, the baseline data of the study of Hayashino et al. had lower SUA $(5.2 \mathrm{mg} / \mathrm{dL})$, higher eGFR $\left(77.4 \mathrm{~mL} / \mathrm{min} / 1.73 \mathrm{~m}^{2}\right)$, older age (64.6 years), more males (73.7), less ARB use (32.2\%) and antihyperuricemic drug use (3.5\%), comparing with ours.

Besides, a study of Chang et al. for a mean of 4.6-year follow-up period with 2367 T2DM participants classified participants into stable $(47.9 \%)$, progression $(20.6 \%)$ or regression (31.5\%) groups based on their change in CKD stage. Their result suggested that the regression group showed the quartile of lowest SUA level $(5.4 \pm 1.5 \mathrm{mg} / \mathrm{dL})$, and the progression group contained the highest SUA level $(6.9 \pm 1.8 \mathrm{mg} / \mathrm{dL})$. Furthermore, they indicated that the SUA level was an independent factor related to the progression of CKD when it was more than $6.3 \mathrm{mg} / \mathrm{dL}$. A lower SUA level could be effective for CKD improvement in patients with T2DM and stage 3-5 CKD, which might indicate that high-normal SUA level might be an extra marker for CKD progression in T2DM patients. The baseline data of the study of Chang et al. had both CKD1-3 and CKD3-5 to estimate the progression and regression

This work is licensed under a Creative Commons Attribution-NonCommercial 4.0 International License. 
Table 3 Associations between serum uric acid and renal outcomes.

\begin{tabular}{|c|c|c|c|c|c|}
\hline & \multicolumn{4}{|c|}{ Serum uric acid concentration quartiles } & \multirow[b]{2}{*}{$P$ value } \\
\hline & 1Q & $2 \mathrm{Q}$ & $3 \mathrm{Q}$ & $4 \mathrm{Q}$ & \\
\hline Serum uric acid ( $\mu \mathrm{mol} / \mathrm{L})$ & 286.02 & 358.23 & 405.50 & 499.14 & \\
\hline Interquartile range of uric acid ( $\mu \mathrm{mol} / \mathrm{L})$ & $(263.00-319.00)$ & $(346.00-370.10)$ & $(393.00-417.00)$ & $(453.00-528.90)$ & \\
\hline \multicolumn{6}{|l|}{ Hazard ratio for progression $(95 \% \mathrm{Cl})$} \\
\hline Crude model & Ref. & $0.859(0.564-1.308)$ & $0.872(0.571-1.332)$ & $0.791(0.517-1.211)$ & 0.753 \\
\hline Age- and gender-adjusted model & Ref. & $0.865(0.566-1.322)$ & $0.875(0.572-1.340)$ & $0.777(0.501-1.204)$ & 0.732 \\
\hline Multivariable-adjusted model & Ref. & $1.364(0.621-2.992)$ & $1.518(0.768-3.002)$ & $1.411(0.706-2.821)$ & 0.652 \\
\hline
\end{tabular}

Multivariable-adjusted model: age, sex, eGFR, 24-h UTP, serum albumin, HbA1c, total cholesterol, the use of antihyperuricemic drug, glomerular class, IFTA, interstitial inflammation, and arteriolar hyalinosis.

of renal function. Interestingly, Tanaka et al. published that male T2DM patients who had significant proteinuria might had a risk of sCr doubling while their SUA is higher than $6.3 \mathrm{mg} / \mathrm{dL}$ (22). As mentioned in their discussion, the causal relationship between SUA and the progression and regression of CKD in T2DM patients could not be clearly determined, given their study design.

Notably, several studies had indicated that SUA-lowering treatment using allopurinol decreased the rate of GFR decline $(23,24)$. A 3-year RCT of losartan in persons with T2DM and CKD 3-4 stage (RENAAL) found that patients who experienced an SUA decrease in the first 6 months using losartan had a lower risk of ESRD or sCr doubling in contrast to participants without SUA reduction (25). Nonetheless, an RCT (Controlled trial of Kidney Disease progression From the Inhibition of Xanthine oxidase (CKD-FIX)) randomly assigned 369 participants to the allopurinol group $(n=185)$ or placebo group $(n=184)(9)$. DKD was the primary cause of CKD in $45 \%$ of patients. The patients in the CKD-FIX study were older, had higher SUA and had lower GFR level than in PERL study, failing to prove that allopurinol had a beneficial effect on the declining of kidney function. The role of SUA-lowering therapy in delaying DKD progression had not yet been proven.

Taken together, it is intriguing that the RCT study had failed to show the statistical benefit of allopurinol on renal outcomes, indicating that even when SUA was reduced to lower levels, kidney progression was not improved as expected in T2DM patients. This result could be possibly explained as the predictive role of SUA on renal function losing might not be direct and could be ascribed to the causal correlation of SUA with other features associated with DN, like the metabolic syndrome and insulin resistance (26). It seemed that uric acidlowering treatment might not be as important as other crucial therapies in DN patients without gout or severe cardiovascular diseases.

In our study, no significant association between the SUA level and renal outcome of ESRD in Chinese T2DM and DN patients was found in our study. Besides, the role of SUA-lowering therapy in delaying DN progression and improving ESRD outcome had not yet been proven. Although antihyperuricemic drugs and other drug which might have effect on SUA levels were adjusted, it was hard to prove the association of SUA-lowering treatment and DN progression, due to the lack of the change of SUA and eGFR. Further study was needed to clarify the renal benefit of the SUA-lowering treatment in the therapy of DN.

Some limitations in this study should be noticed. First, patients were registered from a single center and may not represent the Chinese DN patients as a whole, so further multicenter validation in China and external validation in different ethnic populations was needed. The study sample size was moderate as well. Finally, only the baseline SUA level was analyzed.

\section{Conclusion}

Taken together, no significant association between SUA level and renal outcome of ESRD in Chinese patients with biopsy-confirmed DN was found in our study. Besides, the role of SUA-lowering treatment in delaying DN progression and improving ESRD outcome had not yet been proven. Further study was needed to clarify the renal benefit of the SUA-lowering treatment in the treatment of DN.

\section{Supplementary materials}

This is linked to the online version of the paper at https://doi.org/10.1530/ EC-21-0307.

\section{Declaration of interest}

The authors declare that there is no conflict of interest that could be perceived as prejudicing the impartiality of the research reported.

\section{Funding}

This study was supported by the National Natural Science Foundation of China (Grant numbers 81970626 and 81670662); Key Research and Development Project of Sichuan Science and Technology Department (Grant number 19ZDYF1273).

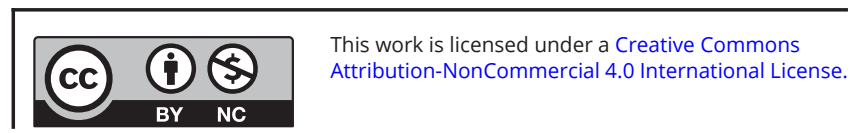




\section{Statement of ethics}

The study protocol was approved by the Institutional Review Board at the West China hospital of Sichuan University (number 2013R01), and writteninformed consent was obtained from all patients.

\section{References}

1 Reutens AT. Epidemiology of diabetic kidney disease. Medical Clinics of North America 201397 1-18. (https://doi.org/10.1016/j. mcna.2012.10.001)

2 Corrigendum to 'Zhang L, Zhao M-H, Zuo L, et al., on behalf of CK-NET Group. China Kidney Disease Network (CK-NET) 2015 Annual Data Report.' Kidney Int Suppl. 2019;9:e1-e81. Kidney International Supplements 202010 e95. (https://doi.org/10.1016/j. kisu.2019.05.001)

3 Kocak MZ, Aktas G, Duman TT, Atak BM \& Savli H. Is uric acid elevation a random finding or a causative agent of diabetic nephropathy? Revista da Associação Médica Brasileira 201965 1155-1160. (https://doi.org/10.1590/1806-9282.65.9.1156)

4 Zoppini G, Targher G, Chonchol M, Ortalda V, Abaterusso C, Pichiri I, Negri C \& Bonora E. Serum uric acid levels and incident chronic kidney disease in patients with type 2 diabetes and preserved kidney function. Diabetes Care 201235 99-104. (https://doi.org/10.2337/dc11-1346)

5 Mauer M \& Doria A. Uric acid and risk of diabetic kidney disease. Journal of Nephrology 202033 995-999. (https://doi.org/10.1007/ s40620-020-00796-z)

6 Hayashino Y, Okamura S, Tsujii S \& Ishii H. Association of serum uric acid levels with the risk of development or progression of albuminuria among Japanese patients with type 2 diabetes: a prospective cohort study [Diabetes Distress and Care Registry at Tenri (DDCRT 10)]. Acta Diabetologica 201653 599-607. (https://doi.org/10.1007/s00592-0150825-X)

7 Chang YH, Lei CC, Lin KC, Chang DM, Hsieh CH \& Lee YJ. Serum uric acid level as an indicator for CKD regression and progression in patients with type 2 diabetes mellitus-a 4.6-year cohort study. Diabetes/Metabolism Research and Reviews 201632 557-564. (https:// doi.org/10.1002/dmrr.2768)

8 Sato Y, Feig DI, Stack AG, Kang DH, Lanaspa MA, Ejaz AA, SánchezLozada LG, Kuwabara M, Borghi C \& Johnson RJ. The case for uric acid-lowering treatment in patients with hyperuricaemia and CKD. Nature Reviews: Nephrology 201915 767-775. (https://doi.org/10.1038/ s41581-019-0174-z)

9 Badve SV, Pascoe EM, Tiku A, Boudville N, Brown FG, Cass A, Clarke P, Dalbeth N, Day RO, de Zoysa JR, et al. Effects of allopurinol on the progression of chronic kidney disease. New England Journal of Medicine 2020382 2504-2513. (https://doi.org/10.1056/NEJMoa1915833)

10 Pinsker JE, Shank T, Dassau E \& Kerr D. Comment on American Diabetes Association. Approaches to glycemic treatment. Sec. 7. In Standards of Medical Care in Diabetes-2015. Diabetes Care 2015;38(Suppl. 1):S41-S48. Diabetes Care 201538 e174. (https://doi. org/10.2337/dc15-0839)

11 An Y, Xu F, Le W, Ge Y, Zhou M, Chen H, Zeng C, Zhang H \& Liu Z. Renal histologic changes and the outcome in patients with diabetic nephropathy. Nephrology, Dialysis, Transplantation 201530 257-266.

12 Tervaert TW, Mooyaart AL, Amann K, Cohen AH, Cook HT, Drachenberg CB, Ferrario F, Fogo AB, Haas M, de Heer E, et al. Pathologic classification of diabetic nephropathy. Journal of the American Society of Nephrology 201021 556-563. (https://doi. org/10.1681/ASN.2010010010) (https://doi.org/10.1093/ndt/gfu250)

13 Sun L, Shang J, Xiao J \& Zhao Z. Development and validation of a predictive model for end-stage renal disease risk in patients with diabetic nephropathy confirmed by renal biopsy. PeerJ 20208 e8499. (https://doi.org/10.7717/peerj.8499)

14 Xiang H, Zhang H, Zhou M, Jiang S, Zhang L, Chen D \& Liu Z. Phosphorus is an independent risk factor for the progression of diabetic nephropathy. Advances in Clinical and Experimental Medicine 201827 1239-1245. (https://doi.org/10.17219/acem/70094)

15 Krolewski AS, Skupien J, Rossing P \& Warram JH. Fast renal decline to end-stage renal disease: an unrecognized feature of nephropathy in diabetes. Kidney International 201791 1300-1311. (https://doi. org/10.1016/j.kint.2016.10.046)

16 Ueno S, Hamada T, Taniguchi S, Ohtani N, Miyazaki S, Mizuta E, Ohtahara A, Ogino K, Yoshida A, Kuwabara M, et al. Effect of antihypertensive drugs on uric acid metabolism in patients with hypertension: cross-sectional cohort study. Drug Research 201666 628-632. (https://doi.org/10.1055/s-0042-113183)

17 Derosa G, Maffioli P, Reiner Ž, Simental-Mendía LE \& Sahebkar A. Impact of statin therapy on plasma uric acid concentrations: a systematic review and meta-analysis. Drugs 201676 947-956. (https:// doi.org/10.1007/s40265-016-0591-2)

18 Ames BN, Cathcart R, Schwiers E \& Hochstein P. Uric acid provides an antioxidant defense in humans against oxidant- and radical-caused aging and cancer: a hypothesis. PNAS 198178 6858-6862. (https://doi. org/10.1073/pnas.78.11.6858)

19 Hayashino Y, Okamura S, Tsujii S \& Ishii H. Association of serum uric acid levels with the risk of development or progression of albuminuria among Japanese patients with type 2 diabetes: a prospective cohort study [Diabetes Distress and Care Registry at Tenri (DDCRT 10)]. Acta Diabetologica 201653 599-607. (https://doi.org/10.1007/s00592-0150825-x)

20 Komendarek-Kowalska M. The assessment of renal function in patients with newly diagnosed hypertension - the role of hyperuricemia as a risk factor for chronic kidney disease - preliminary study. Polski Merkuriusz Lekarski 201742 193-196.

21 Mortada I. Hyperuricemia, type 2 diabetes mellitus, and hypertension: an emerging association. Current Hypertension Reports 20171969. (https://doi.org/10.1007/s11906-017-0770-x)

22 Tanaka K, Hara S, Hattori M, Sakai K, Onishi Y, Yoshida Y, Kawazu S \& Kushiyama A. Role of elevated serum uric acid levels at the onset of overt nephropathy in the risk for renal function decline in patients with type 2 diabetes. Journal of Diabetes Investigation 2015 698-104. (https://doi.org/10.1111/jdi.12243)

23 Siu YP, Leung KT, Tong MK \& Kwan TH. Use of allopurinol in slowing the progression of renal disease through its ability to lower serum uric acid level. American Journal of Kidney Diseases 200647 51-59. (https:// doi.org/10.1053/j.ajkd.2005.10.006)

24 Bartáková V, Kuricová K, Pácal L, Nová Z, Dvořáková V, Švrčková M, Malúšková D, Svobodová I, Řehořová J, Svojanovský J, et al. Hyperuricemia contributes to the faster progression of diabetic kidney disease in type 2 diabetes mellitus. Journal of Diabetes and its Complications 201630 1300-1307. (https://doi.org/10.1016/j.jdiacomp.2016.06.002)

25 Miao Y, Ottenbros SA, Laverman GD, Brenner BM, Cooper ME, Parving HH, Grobbee DE, Shahinfar S, de Zeeuw D \& Lambers Heerspink HJ. Effect of a reduction in uric acid on renal outcomes during losartan treatment: a post hoc analysis of the reduction of endpoints in non-insulin-dependent diabetes mellitus with the angiotensin II Antagonist Losartan Trial. Hypertension 201158 2-7. (https://doi.org/10.1161/HYPERTENSIONAHA.111.171488)

26 Wang H, Zhang H, Sun L \& Guo W. Roles of hyperuricemia in metabolic syndrome and cardiac-kidney-vascular system diseases. American Journal of Translational Research 201810 2749-2763.

Received in final form 7 September 2021

Accepted 15 September 2021

Accepted Manuscript published online 15 September 2021
This work is licensed under a Creative Commons Attribution-NonCommercial 4.0 International License. 